Draft Version September 25, 2018

Preprint typeset using $\mathrm{L}_{\mathrm{T}} \mathrm{E} \mathrm{X}$ style emulateapj v. 2/16/10

\title{
JUPITER WILL BECOME A HOT JUPITER: \\ CONSEQUENCES OF POST-MAIN-SEQUENCE STELLAR EVOLUTION ON GAS GIANT PLANETS
}

\author{
David S. Spiegel ${ }^{1}$, NikKu Madhusudhan ${ }^{2}$ \\ ${ }^{1}$ Astrophysics Department, Institute for Advanced Study, Princeton, NJ 08540 and \\ ${ }^{2}$ Department of Physics and Department of Astronomy, Yale University, New Haven, CT 06511 \\ Draft version September 25, 2018
}

\begin{abstract}
When the Sun ascends the red giant branch (RGB), its luminosity will increase and all the planets will receive much greater irradiation than they do now. Jupiter, in particular, might end up more highly irradiated than the hot Neptune GJ 436b and, hence, could appropriately be termed a "hot Jupiter." When their stars go through the RGB or asymptotic giant branch (AGB) stages, many of the currently known Jupiter-mass planets in several-AU orbits will receive levels of irradiation comparable to the hot Jupiters, which will transiently increase their atmospheric temperatures to $\sim 1000 \mathrm{~K}$ or more. Furthermore, massive planets around post-main-sequence stars could accrete a non-negligible amount of material from the enhanced stellar winds, thereby significantly altering their atmospheric chemistry as well as causing a significant accretion luminosity during the epochs of most intense stellar mass loss. Future generations of infrared observatories might be able to probe the thermal and chemical structure of such hot Jupiters' atmospheres. Finally, we argue that, unlike their main-sequence analogs (whose zonal winds are thought to be organized in only a few broad, planetary-scale jets), red-giant hot Jupiters should have multiple, narrow jets of zonal winds and efficient day-night redistribution.
\end{abstract}

Subject headings: planets and satellites: Jupiter — Sun: evolution — planetary systems radiative transfer — stars: evolution — stars: AGB and post-AGB

\section{INTRODUCTION}

The "hot Jupiter" class of exoplanets was not generally anticipated prior to the discoveries of the first planets around main sequence stars (Mavor \& Queloz 1995; Marcy \& Butler 1996). Their existence, however, could have been predicted long before then, since the Sun's luminosity will increase by a factor of several thousand as it ascends the red giant branch (RGB), thereby turning Jupiter into a hot Jupiter in several billion years.

Roughly $20 \%$ of the more than 700 currently known exoplanet: 1 have masses greater than half of Jupiter's, orbital radii greater than $1 \mathrm{AU}$, and will become hot Jupiters (i.e., for the present purposes, this means they will receive at least as much irradiation as the hot Neptune GJ 436b) before the end of stars' lives. Depending on the efficiency of tidal dissipation in RGB and AGB stars, many of these planets might eventually be tidally engulfed by their

dave@ias.edu,Nikku.Madhusudhan@yale.edu

1 See http://exoplanet.eu (Schneider et al.|2011), or see http://exoplanets.org (Wright et al. 2011) for a differently vetted list. stars (Carlberg et al. 2009; Villaver \& Livio 2009; Nordhaus et al. 2010), where they could play a role in shaping planetary nebulae (PNe) (Soker et al. 1984; Nordhaus \& Blackman 2006; Nordhaus et al. 2007) or creating highly magnetized white dwarfs (Nordhaus et al. 2011). Regardless of whether these planets are eventually swallowed by their stars, at some point in their futures they will be highly irradiated.

Some searches for evidence of planets and other companions to post-main-sequence stars have already been undertaken. White dwarf atmospheres polluted by tidally shredded asteroids indicate the presence of distant planetary companions around $\sim 30 \%$ of white dwarfs (Zuckerman et al. 2010). A post-common-envelope $50-M_{J}$ (Jupiter-mass) object was found in a tight orbit around a white dwarf (Maxted et al. 2006). Tentative evidence has also suggested the existence of several other systems, including two small planets in tight orbits around a subdwarf star (Charpinet et al. 2011), and a jovian body around the pulsating white dwarf GD-66 (Mullally et al. 2007, 2008, 2009) - although recent data have complicated the planetary hypothesis for the latter system (Farihi et al. 2012; Hermes, 


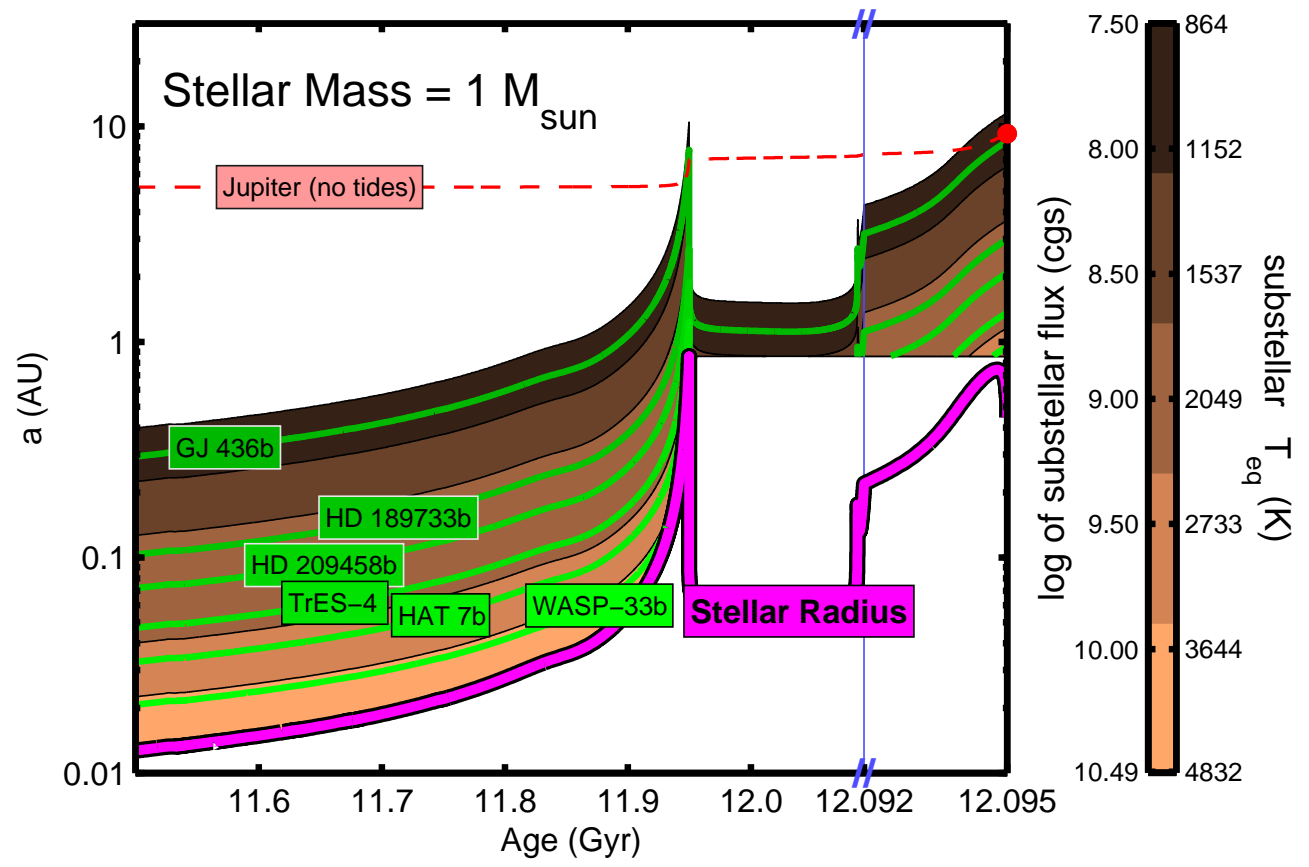

FIG. 1.- Orbital separations where red-giant hot Jupiters can be found around a solar-type star. The evolution of a 1- $M_{\odot}$ star's radius (magenta curve) is shown as a function of age in gigayears (Gyr), and the consequent levels of irradiation as a function of stellar age and orbital distance (color scale). Green curves show regions of (age, orbital radius) space where an object would receive the same incident irradiation as known various known highly irradiated objects: GJ 436b, HD 189733b, HD 209458b, TrES-4, HAT-P-7b, and WASP-33b. The red dashed curve shows how Jupiter's orbit will evolve purely under the influence of stellar mass loss, with a red dot at the end showing the position achieved at the end of our stellar model. Note that, in order to more clearly show the final, very brief part of the star's evolution, the time axis is stretched by a factor of 40 during the ascent of the AGB phase (from $12.092 \mathrm{Gyr}$ until the end of the model). The location where the time axis changes scale is marked with a thin blue line and hatch marks along the horizontal axis.

private communication). Direct-imaging searches for warm companions to white dwarfs have yet to find any (Hogan et al. 2009), but have not yet surveyed large numbers of stars. Finally, Johnson et al. (2011) has found a number of giant planets around slightly evolved, subgiant stars (and some of these planets will soon become hot Jupiters).

Here, we consider the properties of "red-giant hot Jupiters" (RGHJs), a term that we use somewhat loosely to refer generally to hot Jupiters around post-main-sequence stars that are on longer period orbits than their cousins around mainsequence-star. In 92 , we calculate the range of orbital distances at which a gas giant planet around a post-main-sequence star might be considered a hot Jupiter, and we consider possible heating of a planet's bulk interior. In 93 , we examine how the accretion of stellar wind (and perhaps rocky material) onto a RGHJ might pollute its atmosphere. In \$4. we describe changes in Jupiter's chemistry and spectrum that will occur as the Sun evolves beyond the main sequence. In 95 , we describe some qualitative differences between the wind patterns expected on RGHJs and on main-sequence hot Jupiters. In 96. we conclude and speculate on the observability of RGHJs.

\section{BECOMING HOT}

In order to evaluate where around a post-mainsequence star the irradiation is sufficient to produce a hot Jupiter, we use stellar evolution models with zero-age main sequence (ZAMS) masses ranging from 1 to $3 M_{\odot}$. Our stellar models are calculated using the "Evolve Zero-age Main Sequence (EZ) code" (Paxton 2004). For each initial stellar mass, we calculate the star's evolution through the main sequence and to the end of the AGB phase, using metallicity $Z=0.02$.

Figure 1 shows where red-giant hot Jupiters can be found as a function of age and orbital separation, around a $1-M_{\odot}$ star. The evolution of a solarmass star's radius and luminosity by showing where and when a companion would be irradiated similarly to various known hot Jupiters. The evolution of Jupiter's orbit is shown, taking into account only stellar mass loss (which makes the orbit expand as $M_{*}^{-1}$ ) and neglecting tidal interactions between planet and star (which would generally tend to make the orbit shrink).

Figure 2 shows the maximum distance at which a RGHJ could be found around stars ranging from 1 to 3 times the mass of the Sun, at the time 

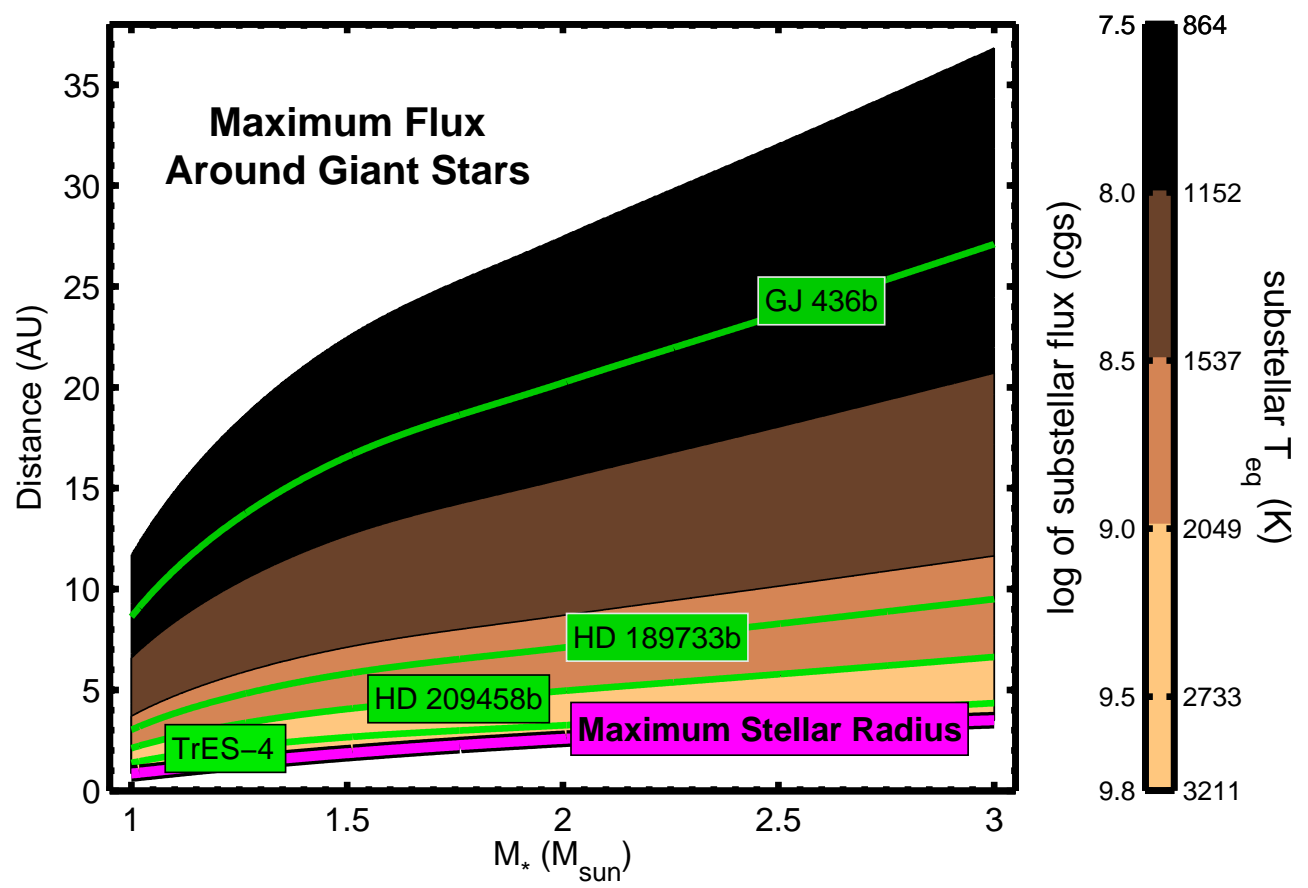

FIG. 2.- Maximum orbital separations where red-giant hot Jupiters can be found around $1-3-M_{\odot}$ stars. The level of irradiation is shown, as a function of orbital distance and stellar mass, at the time of a star's maximum luminosity. Green curves show regions of (stellar mass, orbital radius) space where an object would receive the same irradiation as several highly irradiated planets.

when the stars will be maximally luminous. A companion could (briefly) receive irradiation of $\sim 3 \times$

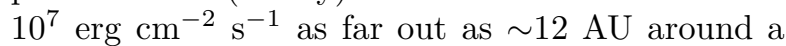
ZAMS $1-M_{\odot}$ star (during the RGB phase) or as far out as $\sim 35 \mathrm{AU}$ around a $3-M_{\odot}$ star (during the AGB phase).

The detailed shape of the shaded "hot" contours in Figs. 1 and 2 depends on properties of stars (including metallicity and mass loss) that are surely not perfectly captured by our models, but the qualitative features of our models should hold for real stars. Planets at a given hot region in either the left or the right panel of this figure could have had main-sequence orbital separations that were either greater or less than the given abscissa - greater if tidal interactions are reducing the orbital separation, less if stellar mass loss is causing it to expand ${ }^{2}$ We note that more post-main-sequence hot Jupiters in a volume-limited survey will be found at moderate separation (one to a few AU) around subgiant and early giant branch stars than at more extreme separation around stars at the tip of the RGB or the AGB, simply because of the relative timescales of the respective stages of stellar evolution.

Finally, a jovian planet that is sufficiently highly

${ }^{2}$ When the star is early on the subgiant branch, its angular rotation rate might not have decreased by much yet, and tidal interactions would actually cause companions whose orbital mean motion is less than the stellar rotation rate to move out (see, e.g., Llovd 2011), similar to the Earth-Moon interaction. irradiated might achieve non-negligible ionization in its atmosphere. Winds crossing the planetary magnetic field could, therefore, induce an electric field that would drive currents in the atmosphere or in the deep interior, similar to an analogous process in main sequence hot Jupiters (Batygin \& Stevenson 2010; Perna et al. 2010a, b; Batygin et al. 2011; Menou 2012). This process might be able to contribute to heating the bulk interior of a planet 3 In this way, Ohmic heating (or any other mechanism that converts irradiation energy into thermal energy in the deep interior), if viable, could "reset" a planet's evolutionary clock, analogously to the process described by Ibgui \& Burrows (2009). The degenerate stellar core that is left at the end of the AGB phase is itself extremely luminous, and might further contribute to heating (and reheating) a planet that remains in the planetary nebula (PN), and Villaver \& Livio (2007) consider possible evaporation of a planet that might occur due to X-ray and ultraviolet irradiation. A giant planet's luminosity is generally thought to be a reasonably good indication of its mass, irrespective of initial conditions ("hot-start," "coldstar," or "warm-start"), so long as the object is sufficiently old and its age is known (Marley et al. 2007; Fortney et al. 2008; Spiegel \& Burrows 2012). However, if RGHJs or post-RGHJs are observed (or-

${ }^{3}$ We note, though, that Wu \& Lithwick (2012) argue that Ohmic dissipation cannot reinflate objects that have already cooled. 
biting giant stars or the remnant white dwarfs, assuming that they did not fully evaporate during the PN phase), it is conceivable that mass estimates based on photometry could be biased high if the object's orbit caused it to be sufficiently highly irradiated and if this potential reheating effect is not taken into account.

However, there is an an important difference between RGHJs and main-sequence hot Jupiters in terms of the timescale during which the object is highly irradiated. Hot Jupiters around main-sequence stars need to convert only a small fraction of incident stellar irradiation to a form that heats the convective interior in order to have a strong influence on the bulk thermal state (Guillot \& Showman 2002; Liu et al. 2008), but this is because the objects can find a quasi-steady thermal state on a timescale of tens of millions of years. But the total energy intercepted by Jupiter during the Sun's RGB and AGB phases is probably insufficient to cause significant reheating, even if the energy were deposited deep in the convective core, simply because Jupiter will not receive extreme irradiation for a long enough time. Planets that are closer to their stars receive greater irradiation, but are at greater risk of being tidally engulfed by their stars (potentially causing observable signatures; Struck-Marcell 1988) and therefore might not survive to the end of the AGB phase. Since many of the most highly irradiated RGHJs will eventually merge with their stars, it is unclear whether any of the objects that are on distant-enough orbits to avoid engulfment (i.e., to end up on the outside of the circum-white-dwarf "gap" predicted by Nordhaus et al. 2010) could be irradiated sufficiently to cause any significant bulk reheating.

\section{ACCRETING MATERIAL}

As stars ascend the RGB and the AGB, they lose mass much faster than they do when on the main sequence. Furthermore, since wind speeds are typically a multiple of the surface escape speed that is of order a few times unity (Willson 2000), giant stars' winds are much slower than those of main sequence stars. For instance, Zuckerman \& Dyck (1989) examined a sample of $\sim 100$ AGB stars and found that their outflow velocities are often less than $40 \mathrm{~km} \mathrm{~s}^{-1}$, and tend to cluster around $5-25 \mathrm{~km} \mathrm{~s}^{-1}$. A massive enough planet could have an escape speed that is greater than the relative speed $v_{\text {rel }}$ between the planet and the wind. In such a case, the planet will accrete the stellar wind material that it passes through, with an effective "accretion radius" that may be found by setting to zero the sum of the specific gravitational and kinetic energies of wind particles, as per Bondi \& Hovle (1944) or Dong et al.
(2010):

$$
R_{\mathrm{acc}}=\frac{2 G M_{p}}{v_{\mathrm{rel}}^{2}}=2 a\left(\frac{M_{p}}{M_{*}}\right)\left(\frac{v_{K}}{v_{\mathrm{rel}}}\right)^{2},
$$

where $M_{p}$ and $M_{*}$ are the respective masses of the planet and the star, and $a$ is the orbital semimajor axis, and $v_{K}$ is the Keplerian orbital speed. In particular, when $R_{\text {acc }} \geq R_{p}$ (where $R_{p}$ is the planet's radius), the planet readily accretes stellar wind 4 When $R_{\text {acc }}<R_{p}$, the situation is significantly more complicated, but even in this case some stellar wind material will surely be intercepted and accreted. For now, we consider planets massive enough that $R_{\text {acc }} \geq R_{p}$, but the qualitative conclusions are not necessarily altered even if this condition is not met.

If the planet accretes everything within $R_{\text {acc }}$, at its relative speed $v_{\text {rel }}$, the accretion rate is

$$
\dot{M}_{p} \sim\left(\pi R_{\text {acc }}^{2}\right) \rho[a] v_{\text {rel }}
$$

where $\rho[a]$ is the wind density at the location of the planet:

$$
\rho[a]=-f_{i} \frac{\dot{M}_{*}}{4 \pi a^{2} v_{w}} .
$$

In Eq. (3), $\dot{M}_{*}$ is the stellar mass-loss rate, $v_{w}$ is the speed of the stellar wind, and $f_{i}$ is the "wind isotropy factor," 1 for a purely isotropic wind and either less than or greater than 1 depending on if the wind is less or more dense at the planet's position than an isotropic wind would be. The planet's accretion rate may, therefore, be expressed as

$$
\begin{aligned}
\dot{M}_{p} & \sim-f_{i} \dot{M}_{*}\left(\frac{R_{\mathrm{acc}}}{2 a}\right)^{2}\left(\frac{v_{\mathrm{rel}}}{v_{w}}\right) \\
& \sim-f_{i} \dot{M}_{*}\left(\frac{M_{p}}{M_{*}}\right)^{2}\left(\frac{v_{K}}{v_{\mathrm{rel}}}\right)^{4}\left(\frac{v_{\mathrm{rel}}}{v_{w}}\right) .
\end{aligned}
$$

To a rough approximation, the total accreted mass is

$$
\Delta M_{p} \sim\left|\Delta M_{*}\right|\left(\frac{M_{p}}{M_{*}}\right)^{2}\left(\frac{f_{i} v_{K}^{4}}{v_{w} v_{\mathrm{rel}}^{3}}\right),
$$

where $\Delta M_{*}$ mass lost by the star.

The accreted mass can make a non-negligible contribution to the mass of the atmosphere already present. The mass, above pressure $P$, of the atmosphere of a planet whose surface gravity is $g$ scales inversely with planet mass:

$$
M_{\mathrm{atm}}[P]=4 \pi R_{p}^{2} \frac{P}{g}=\frac{4 \pi R_{p}^{4} P}{G M_{p}} .
$$

${ }^{4}$ Although some terrestrial planets' atmospheres might be eroded by main sequence stellar winds (Zendejas et al.2010), evolved stars' winds consist of much lower energy particles and will tend to accrete onto jovian-mass planets. 
The ratio of the accreted mass to the mass of the atmosphere above a given pressure level, then, is

$$
\begin{aligned}
\frac{\Delta M_{p}}{M_{\mathrm{atm}}[P]} \sim & \frac{G M_{p}^{3}\left|\Delta M_{*}\right|}{4 \pi R_{p}^{4} P M_{*}^{2}} \times\left(\frac{f_{i} v_{K}^{4}}{v_{w} v_{\mathrm{rel}}^{3}}\right) \\
\sim & 400 \times\left(\frac{M_{p}}{10 M_{J}}\right)^{3}\left(\frac{P}{100 \text { bars }}\right)^{-1} \\
& \times\left(\frac{\left|\Delta M_{*}\right|}{M_{*} / 2}\right)\left(\frac{M_{*}}{M_{\odot}}\right)^{-1} .
\end{aligned}
$$

In eq. (8) we have assumed that $f_{i} \approx 1, R_{p} \approx R_{J}$, and we have taken the velocity-dependent terms (the parenthetical term in eq. (7) to be $\sim 10^{-2}$, the value appropriate for an object at $5 \mathrm{AU}$ around a solar-mass star whose wind is $40 \mathrm{~km} \mathrm{~s}^{-1}$. It is worth emphasizing that this ratio scales with the cube of the planet's mass. Equation (77) shows that massive planets and brown dwarfs could accrete a large multiple of their atmospheric masses, causing atmospheric chemical abundances to be dominated by the abundances of accreted stellar material 5 Although the accreted mass might be large compared with an object's the atmosphere mass, it is a small fraction of the object's total mass - of order $\sim 10^{-5} \times\left(M_{p} / M_{J}\right)^{-2}$ — and has a negligible effect on the orbit (Villaver \& Livio 2009). It is possible that the Bondi-Hoyle accretion rate is an overestimate of the true rate at which a companion would accrete stellar wind, since much of the material inside $R_{\text {acc }}$ but outside $R_{p}$, although formally gravitationally bound to the companion, would have excess angular momentum that must be shed before it is actually incorporated into the companion's atmosphere. Nevertheless, a massive enough companion will encounter an amount of gravitationally bound stellar wind material that is very large compared with the mass of its atmosphere, and it is difficult to avoid the conclusion that this has the capacity to strongly influence the companion's atmospheric composition, especially since the atmosphere of a highly irradiated planet develops a deep radiative layer that is statically stable and might not efficiently mix with the deep interior of the planet (Spiegel et al. 2009).

Figure 3 shows how the pollution of the planet's atmosphere with stellar wind material scales with planet mass, stellar wind outflow velocity, and orbital separation. More massive planets accrete a greater multiple of the already-present atmosphere mass $\left(\propto M_{p}^{3}\right)$, and planet accrete more readily when closer to the primary and when the wind velocities are lower. For a wide range of conditions, the planet accretes more mass than was previously present in the atmosphere at altitudes above the 100-bar iso-

\footnotetext{
5 See Spiegel et al. (2011) regarding the appropriateness of a mass-cut for distinguishing brown dwarfs from giant planets.
}

bar.

The gravitational energy lost by stellar wind material as it falls onto the planet constitutes a power source $\left(\sim G M_{p} \dot{M}_{p} / R_{p}\right)$. Since $\dot{M}_{p}$ is proportional to $M_{p}^{2}$ (see eq. (4), the accretion luminosity $L_{\text {acc }}$ scales with $M_{p}^{3}$ as follows:

$$
\begin{aligned}
L_{\mathrm{acc}} \sim & -\frac{G M_{p} \dot{M}_{*}}{R_{p}}\left(\frac{M_{p}}{M_{*}}\right)^{2}\left(\frac{f_{i} v_{K}^{4}}{v_{w} v_{\mathrm{rel}}}\right) \\
\sim & 10^{29} \mathrm{erg} \mathrm{s}^{-1} \times\left(\frac{\left|\dot{M}_{*}\right|}{10^{-7} M_{\odot} \mathrm{yr}^{-1}}\right) \\
& \times\left(\frac{M_{p}}{10 M_{J}}\right)^{3}\left(\frac{M_{*}}{M_{\odot}}\right)^{-2} .
\end{aligned}
$$

As in eq. (8), we have again taken the velocitydependent terms to be $\sim 10^{-2}$ (and $f_{i} R_{J} / R_{p} \approx 1$ ). The shocked in-falling material achieves a characteristic temperature $T_{\text {acc }}$ found by equating $k_{B} T_{\text {acc }}$ to the energy lost per particle:

$$
\begin{aligned}
T_{\text {acc }} & \sim \frac{G M_{p} m_{p}}{k_{B} R_{p}} \\
& \sim 2 \times 10^{6} \mathrm{~K} \times\left(\frac{M_{p}}{10 M_{J}}\right),
\end{aligned}
$$

where $k_{B}$ is Boltsmann's constant and $m_{p}$ is the proton mass. The accretion process, therefore, creates an optically thin, soft-X-ray-emitting coronal envelope around the planet, which could produce a faint observational signature. If the accretion power is taken to be the X-ray luminosity, then this luminosity peaks at $\sim 10^{29} \mathrm{erg} \mathrm{s}^{-1}$ for the case of a $10-M_{J}$ companion at $5 \mathrm{AU}$ around a $1-M_{\odot}$ (ZAMS) primary. A more massive primary would have a somewhat more luminous accretion shock, with luminosities briefly reaching $\sim 10^{30} \mathrm{erg} \mathrm{s}^{-1}$ for a $10-$ $M_{J}$ companion.

Figure 4 shows the evolution of the accretion power, calculated with eq. (9), in comparison to the intercepted stellar irradiation. This calculation assumes a $1-M_{\odot}$ (ZAMS) primary with a $10-M_{J}$ companion at a range of orbital separations, from 3-10 AU and with a range of assumed wind velocities, from $10-40 \mathrm{~km} \mathrm{~s}^{-1}$. The accretion power exceeds the irradiating power for only a relatively small portion of the post-main-sequence evolution. Since accretion power scales with the cube of planet

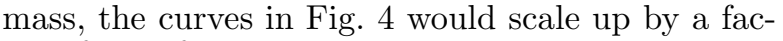
tor of $\sim 30$ for a $30-M_{J}$ companion and down by a factor of 1000 for a $1-M_{J}$ companion (such that for a Jupiter-mass companion, the irradiation power at all times exceeds the accretion power).

An additional mechanism to add material to the planet's atmosphere involves capture of asteroids and planetesimals. Dong et al. (2010) point out 


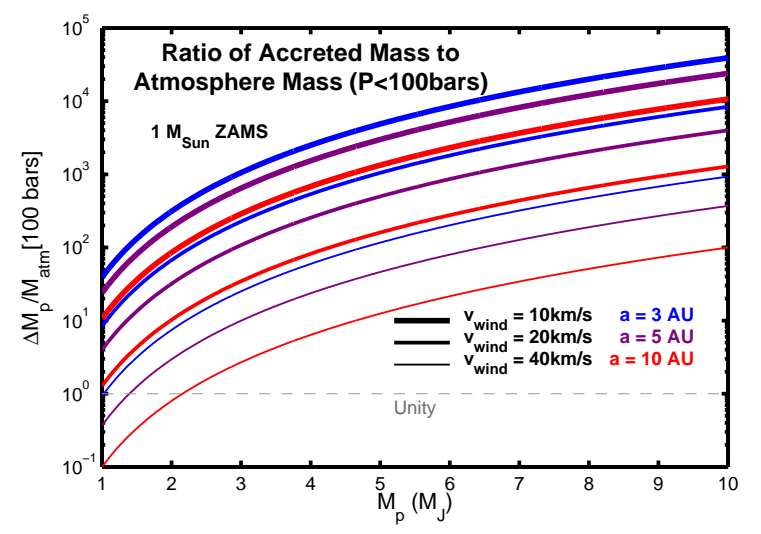

FIG. 3.- Approxiate ratio of accreted mass to atmosphere mass above 100 bars, for a $1 M_{\odot}$ primary. This figure is a visualization of equation (7), for a range of planet masses $\left(1-10 M_{J}\right)$, orbital separations $(3,5$, and $10 \mathrm{AU}$, denoted by colors - blue, purple, and red, respectively), and stellar wind velocities $\left(10,20\right.$, and $40 \mathrm{~km} \mathrm{~s}^{-1}$, denoted by line thicknesses - thick, medium, and thin, respectively). A gray dashed line indicates the ordinate value 1 , above which the mass of accreted material exceeds the mass of the atmosphere that was already present.

that orbiting bodies that are sufficiently small can be dynamically affected by passing through stellar wind material, and can be dragged inward, changing their period ratios with respect to the Jupiter-mass object. If a significant number of these are accreted, this process could affect the bulk or atmospheric metallicity of the giant planet, and its element ratios. Note that changes in orbital eccentricity, of the sort described by Veras et al. (2011), could also contribute to small bodies having RGHJ-orbit-crossing trajectories. Furthermore, in the case of a binary star system, Kratter \& perets (2012) show that orbital instabilities can cause a planet to migrate significantly, even to "hop" from one star to the other, which could again lead to significant accretion of small rocky or icy material.

An evolved star that has gone through third dredge-up can have significantly altered atmospheric abundances (Kippenhahn \& Weigert 1990), and a $\mathrm{C} / \mathrm{O}$ ratio greater than unity even if on the main sequence its $\mathrm{C} / \mathrm{O}$ ratio had been near solar ( 0.5; Asplund et al. 2009) 6 A planet accreting such a star's wind, therefore, could appear to be a carbon-enriched planet 7 Madhusudhan et al. (2011a) found that multi-band infrared photometry of WASP-12b (Hebb et al. 2009) suggests at high confidence that this planet has a $\mathrm{C} / \mathrm{O}$ ratio of 1 or greater, and Madhusudhan et al. (2011b)

${ }^{6}$ Le Bertre et al. (2001) show that $\sim 50 \%$ of mass loss from AGB stars is carbon-enriched.

7 The planet's upper atmosphere might continue to remain carbon-enriched for as much as tens of millions of years after the end of the AGB phase (perhaps much less, depending on the strength of atmospheric mixing), after which the atmosphere will become well mixed with the deep interior and will no longer retain the memory of stellar wind pollution.

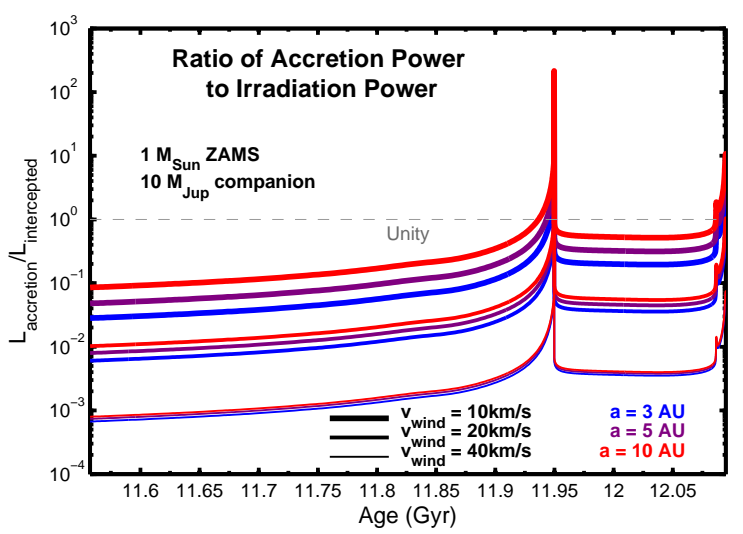

FIG. 4.- Ratio of the accretion power to the intercepted stellar irradiation, for a $10-M_{J}$ planet around a $1 M_{\odot}$ primary. This figure is a visualization of equation (9), for a range of orbital separations $(3,5$, and $10 \mathrm{AU}$, denoted by colors - blue, purple, and red, respectively), and stellar wind velocities $\left(10,20\right.$, and $40 \mathrm{~km} \mathrm{~s}^{-1}$, denoted by line thicknesses - thick, medium, and thin, respectively). A gray dashed line indicates the ordinate value 1 , above which the accretion power exceeds the irradiating power. This occurs for only a narrow time window, outside of which the accretion power is only a small perturbation to the total heating of the planet. and Oberg et al. (2011) explored formation conditions that are necessary to form a carbon-rich hot Jupiter around a main sequence star. Here, we have shown that a massive planet around an evolved star could acquire a carbon-enriched atmosphere, even if the bulk interior of the planet has different chemical abundances. We note that a similar process might act to change the composition of low-mass stellar companions (e.g., M stars or white dwarfs) of AGB stars, as well (Livio \& Warner 1984; Farihi et al. 2010).

\section{CHEMICAL AND SPECTRAL SIGNATURES}

The changes in the incident irradiation experienced by a Jupiter around an evolving star cause distinct changes in chemical and thermal properties in the planetary atmosphere that can be observed in spectra. Here, we investigate the changes in chemistry and emergent spectra of Jupiter as the Sun approaches the RGB phase. We model the diskintegrated emergent spectra of Jupiter at various effective temperatures using the radiative transfer code of Madhusudhan \& Seager (2009). The code solves line-by-line radiative transfer in a 1-D planeparallel atmosphere under the assumption of hydrostatic equilibrium, local thermodynamic equilibrium (LTE), and chemical equilibrium. For each planetary effective temperature we consider, we adopt a 1-D averaged thermal profile of the planetary atmosphere based on the analytic model of Guillot (2010). We choose model parameters for the thermal profile such that its thermal gradient matches those of thermal profiles retrieved for exoplanets with similar irradiation levels (e.g. GJ 436b; Madhusudhan \& Seager 2011). We consider all the 


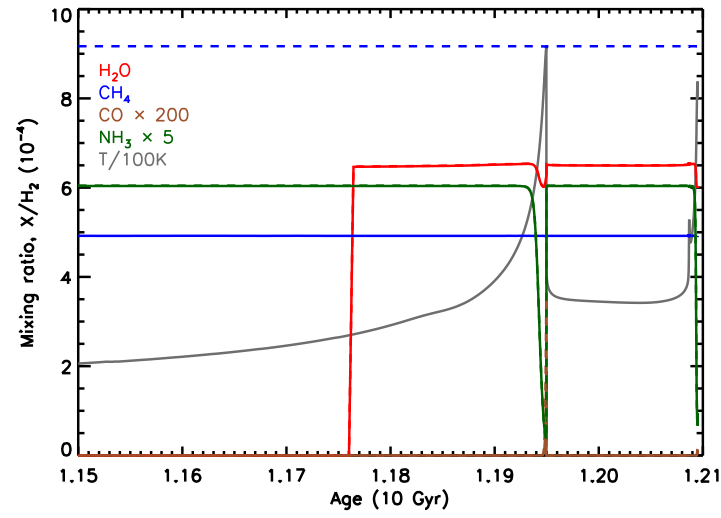

FIG. 5.- Post-main-sequence evolution of Jupiter's equilibrium temperature and atmospheric chemical composition. The temperature evolution is shown in gray (the gray curve is the planet's equilibrium temperature divided by $100 \mathrm{~K}$, assuming that the star follows our adopted solar evolution model), and water, methane, carbon monoxide, and amonia mixing ratios are shown in red, blue, brown, and green, respectively. Solid/dashed curves indicate abundances assuming carbon-to-oxygen ratio of $0.5 / 1$. These alternate $\mathrm{C} / \mathrm{O}$ ratios illustrate possible changes in chemistry as a result of post-AGB atmospheric pollution or simply what might be found for planets with different primordial $\mathrm{C} / \mathrm{O}$ ratios. The main effect of doubling the $\mathrm{C} / \mathrm{O}$ ratio is simply to double the $\mathrm{CH}_{4}$ abundance. The evolution of the atmospheric water abundance is dramatic (and nearly independent of $\mathrm{C} / \mathrm{O}$ ratio), from undetectable levels (as at present) to perhaps several times $10^{-4}$ (depending on the as-yet unknown oxygen content of Jupiter) after the equilibrium temperature exceeds the condensation point of water. The dominant carbonbearing species $\left(\mathrm{CH}_{4}\right.$ and $\left.\mathrm{CO}\right)$ exhibit negligible temporal evolution in chemical equilibrium.

major sources of opacity prevalent in $\mathrm{H}_{2}$-dominated atmospheres, namely, line absorption due to $\mathrm{H}_{2} \mathrm{O}$, $\mathrm{CO}, \mathrm{CH}_{4}, \mathrm{CO}_{2}$, and $\mathrm{NH}_{3}$, and continuum opacity due to $\mathrm{H}_{2}-\mathrm{H}_{2}$ collision-induced absorption. For a given thermal profile, we compute the mixing ratio profiles of the molecular species under the assumption of chemical equilibrium (Burrows \& Sharp 1999). We also compute the equilibrium concentrations of the various species over a wide range of planetary age, and hence thermal conditions, using the equilibrium chemistry code developed in Seager et al. (2005), and updated/used in several following works (e.g. Miller-Ricci et al. 2009; Madhusudhan \& Seager 2011).

The changes in the chemical composition of the jovian atmosphere with stellar age are shown in Fig. 5. We discuss the evolution of the molecular mixing ratios of the dominant species $\left(\mathrm{H}_{2} \mathrm{O}, \mathrm{CO}\right.$, $\mathrm{CH}_{4}$, and $\mathrm{NH}_{3}$ ) in chemical equilibrium at a nominal pressure of 1 bar. The changes in chemistry are driven largely by the changing temperature of the atmosphere.

An important consequence is the enhanced observability of $\mathrm{H}_{2} \mathrm{O}$ after a stellar age $(t)$ of $\sim 11.76$ Gyr (i.e., after the equilibrium temperature exceeds the sublimation point of water ice). At present, the $\mathrm{H}_{2} \mathrm{O}$ abundance in the jovian atmosphere is unknown. The low temperatures $\left(T_{\text {eff }} \sim\right.$ $125 \mathrm{~K}$ ) cause $\mathrm{H}_{2} \mathrm{O}$ to condense out to the deep layers $\left(P \gtrsim 10\right.$ bar) of the atmosphere, making $\mathrm{H}_{2} \mathrm{O}$ inaccessible to spectroscopic observations (Atreya 2004). As the temperature increases beyond the condensation temperature of $\mathrm{H}_{2} \mathrm{O}$, at $\sim 11.76 \mathrm{Gyr}$, gaseous $\mathrm{H}_{2} \mathrm{O}$ becomes abundant in the atmosphere and hence would be observable in spectra from nearEarth space telescopes. $\mathrm{H}_{2} \mathrm{O}$ then continues to be the dominant oxygen-bearing molecule for the rest of the stellar lifetime 8 A slight decrement in the $\mathrm{H}_{2} \mathrm{O}$ abundance is observed briefly as the star ascends the RGB $(t \sim 11.95 \mathrm{Gyr})$, when the planetary temperature exceeds $\sim 600 \mathrm{~K}$; at such temperatures, part of the oxygen $(\lesssim 10 \%)$ is bound in silicates.

As for carbon-bearing species, most of the carbon in the jovian atmosphere continues to be in the form of $\mathrm{CH}_{4}$, as is presently known (Atreva 2004; Karkoschka \& Tomasko 2010). In chemical equilibrium, CO is predicted to be negligible for most of the stellar lifetime, and reaches a few ppm only at the peak temperatures $(\sim 900 \mathrm{~K})$ when the star enters the RGB phase. However, our CO estimate is only a lower limit, since it is known that nonequilibrium chemistry in the form of vertical eddy diffusion can cause enhancements in the $\mathrm{CO}$ abundance by over two orders of magnitude, depending on the strength of large-scale vertical mixing (Hubenv \& Burrows 2007; Madhusudhan \& Seager 2011; Visscher \& Moses 2011). In late stages of the evolution, substantial accretion of stellar carbonrich material onto the planetary atmosphere is expected to enhance the $\mathrm{C} / \mathrm{O}$ ratio of the planet. If present-day Jupiter is oxygen-rich, with $\mathrm{C} / \mathrm{O}=0.5$, such as the Sun, a jovian atmosphere around an AGB star that has gone through third dredge-up (see 33) can potentially have a $\mathrm{C} / \mathrm{O} \geq 1$, making it a Carbon-rich Planet (CRP; Madhusudhan et al. 2011b). As shown in Fig. 5, such an increase in the $\mathrm{C} / \mathrm{O}$ ratio, due to increased carbon, causes an increased $\mathrm{CH}_{4}$ composition, while the consequences on the remaining species remain minimal at the temperatures under consideration?

The changes in chemistry and thermal irradiation cause concomitant signatures in the emergent spectra of the jovian atmosphere at various stages of the Sun's evolution. Figure [6] shows emergent spectra at 8 different effective temperatures, corresponding to different solar ages. At very low temperatures $\left(T_{\text {eff }} \lesssim 300 \mathrm{~K}\right)$, absorption features of

${ }^{8}$ For temperatures of $T \sim 200-900 \mathrm{~K}$ that are relevant in this context, $\mathrm{H}_{2} \mathrm{O}$ is the dominant oxygen-bearing molecule and $\mathrm{CH}_{4}$ is the dominant carbon-bearing molecule in $\mathrm{H}_{2}$ dominated atmospheres, irrespective of the $\mathrm{C} / \mathrm{O}$ ratio (e.g. Madhusudhan et al. 2011b).

9 The chemical consequences of high $\mathrm{C} / \mathrm{O}$ ratios are more readily apparent, e.g. via a substantially decreased $\mathrm{H}_{2} \mathrm{O}$ abundance, at $T \gtrsim 1200 \mathrm{~K}$ (Madhusudhan et al. 2011b). 


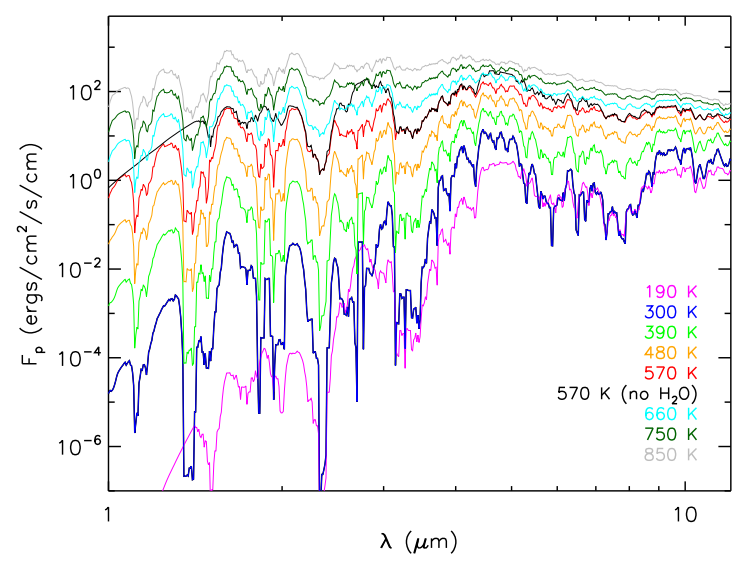

FIG. 6. - Example spectra of Jupiter as a function of equilibrium temperature, as seen from a distance of 5 AU. As Jupiter heats up, it becomes brighter by orders of magnitude in the near infrared part of the spectrum. Methane features dominate the spectra at very low temperatures, and water features become prominent as the equilibrium temperature exceeds the sublimation point of water ice. The red and the black curves are for the same equilibrium temperature $(570 \mathrm{~K})$, with and without $\mathrm{H}_{2} \mathrm{O}$, respectively. The difference between them clearly indicates the spectral influence of atmospheric $\mathrm{H}_{2} \mathrm{O}$ in the near infrared.

methane dominate the spectrum, and water vapor is non-existent. As the jovian atmosphere continues to be heated with increasing insolation, the blackbody continuum is enhanced in the emergent spectrum. At the same time, features of water vapor become conspicuous as atmospheric temperatures exceed $\sim 300 \mathrm{~K}$. For the rest of the solar evolution, features of $\mathrm{H}_{2} \mathrm{O}$ and $\mathrm{CH}_{4}$ dominate the spectrum. Consequently, in the distant future $(t \gtrsim 11.8 \mathrm{Gyr})$, space telescopes will be able to derive the abundance of water vapor in the jovian atmosphere from infrared spectra $1 \mathrm{O}: \mathrm{M}$ While the $\mathrm{H}_{2} \mathrm{O}$ and $\mathrm{CH}_{4}$ abundances so derived from spectra would provide estimates of the $\mathrm{C} / \mathrm{H}$ and $\mathrm{O} / \mathrm{H}$ elemental abundance ratios, a distinctive determination of whether the atmospheric $\mathrm{C} / \mathrm{O}$ ratio exceeds unity (and hence is carbon-rich; Madhusudhan et al. 2011b) would require that the $\mathrm{C} / \mathrm{H}$ and $\mathrm{O} / \mathrm{H}$ ratios be estimated to precisions better than a factor of $\sim 2$. The curves in Fig. 6] represent spectra as seen from a distance of $5 \mathrm{AU}$, and may be scaled to represent fluxes from a hypothetical post-main-sequence Jupiter analog around a star at any distance $d$ from us by multiplying the values by $5.9 \times 10^{-10}(d / 1 \mathrm{pc})^{-2}$.

\section{ATMOSPHERIC MOTIONS}

10 At present, water vapor is under-abundant in the jovian atmosphere and hence not observable in infrared spectra. The Juno mission (Bolton \& Bolton 2010) currently en route to Jupiter is expected to measure the water abundance in Jupiter using microwave sounding.

11 At this distant-future epoch, of course, the Earth will be uninhabitable by life as we know it.
The Rhines length scale is a measure of the typical size of zonal bands of winds in a planetary atmosphere (Showman et al. 2010; Vasavada \& Showman 2005). This length scale, which describes the boundary between turbulent (small) scales and the scales at which coherent Rossby waves dominate, depends on flow speed $U$ as follows: $L_{\mathrm{Rh}}[\beta] \sim \pi(U / \beta)^{1 / 2}$, where $\beta$ is the northward derivative of the Coriolois parameter $(f \equiv 2 \Omega \sin [$ latitude]; and $\Omega$ is the planetary angular rotation rate). This length may be rewritten as an angular scale by dividing by the planet's radius, yielding an equatorial Rhines angle that may be expressed as

$$
\theta_{\mathrm{Rh}} \sim 18^{\circ}\left(\frac{U}{100 \mathrm{~m} / \mathrm{s}} \times \frac{P_{\mathrm{rot}}}{1 \text { day }}\right)^{1 / 2}\left(\frac{R_{p}}{R_{J}}\right)^{-1 / 2} .
$$

Since post-main-sequence stellar evolution does nothing to increase the strength of the stellar tide raised on a planet, RGHJs at separations of $\sim 1 \mathrm{AU}$ or more are not tidally locked to their stars, and rotate at the same angular frequency they had during the main-sequence portions of their stars' lives. Their day lengths might be $\sim 10 \mathrm{hrs}$ if extrasolar Jupiters are similar to Jupiter and Saturn, or perhaps even significantly less if they spin as fast as the $\sim 2-3$-hr periods that have been inferred for some brown dwarfs (Clarke et al. 2002; Artigau et al. 2009). We note that, lacking permanent day and night, RGHJs probably have more uniform atmospheric temperature distributions than their tidally locked bretheren around main-sequence stars, and their heat redistribution parameter $P_{n}$ (describing the portion of day-side incident flux that is re-radiated from the night side, per Burrows et al. 2006) might be very nearly 0.5 .

It is difficult to know exactly how fast typical wind speeds on RGHJs are likely to be, but it seems reasonable to assume they would be between the speeds of winds on Jupiter $\left(\sim 40 \mathrm{~m} \mathrm{~s}^{-1}\right)$ and those on main-sequence hot Jupiters. Showman et al. (2010) and Menou (2012) suggest two approximate scaling relations for how hot Jupiter zonal wind speeds depend on day-night temperature difference $\Delta T$. If $v_{\text {rot,eq }} \equiv \Omega_{p} R_{p}$ is the equatorial rotation speed, then these two scaling relations can be written as

$$
U_{1} \sim\left(\frac{k_{B}}{\mu m_{p}} N_{\mathrm{SH}} \Delta T\right)^{1 / 2} \text { and } \quad U_{2} \sim \frac{U_{1}^{2}}{v_{\text {rot,eq }}},
$$

where $\mu$ is mean molecular weight in units of the proton mass and $N_{\mathrm{SH}}$ is the number of scale heights over which there is a vertical wind shear of $U_{1}$ or $U_{2}$, respectively 12 For a hot Jupiter,

12 Both expressions in Eq. (14) are found in Showman et al. (2010) and the latter (for " $U_{2}$ ") is found in Menou (2012). 
TABLE 1

Scale of Atmospheric Features

\begin{tabular}{l|cccc}
\hline \hline Object & $\begin{array}{c}U \\
\left(\mathrm{~m} \mathrm{~s}^{-1}\right)\end{array}$ & $\begin{array}{c}P_{\mathrm{rot}} \\
\text { (days) }\end{array}$ & $\begin{array}{c}R_{p} \\
\left(R_{J}\right)\end{array}$ & $\theta_{\mathrm{Rh}}$ \\
\hline Jupiter & $\sim 40$ & 0.4 & 1 & $\sim 7^{\circ}$ \\
Hot Jupiter & $\sim 10^{3}$ & 4 & 1.3 & $\sim 100^{\circ}$ \\
RGHJ & $\sim 10^{2} ?$ & 0.4 & 1 & $\sim 10^{\circ}$
\end{tabular}

Note. - This table presents typical values of windspeed $(U)$, rotation period $\left(P_{\text {rot }}\right)$, planetary radius $\left(R_{p}\right)$, and angular Rhines scale $\left(\theta_{\mathrm{Rh}}\right)$ for Jupiter, for a hot Jupiter around a main-sequence star, and for a red-giant hot Jupiter (RGHJ) that is too far from its star to be tidally locked. The small angular Rhines scale for the RGHJ suggests that it would have narrow-banded jets, similar to Jupiter and different from what dynamical models suggest for ordinary hot Jupiters.

$v_{\text {rot,eq }}, U_{1}$, and $U_{2}$ all are of order one or a few $\mathrm{km} \mathrm{s}^{-1}$. For a RGHJ, $v_{\text {rot,eq }}$ is an order of magnitude greater, so, over $N_{\mathrm{SH}}=3$ scale heights, $U_{1} \sim 1000 \mathrm{~m} \mathrm{~s}^{-1}(\Delta T / 100 \mathrm{~K})^{1 / 2}$, while $U_{2} \sim 100 \mathrm{~m} \mathrm{~s}^{-1}(\Delta T / 100 \mathrm{~K})$.

In Table 1] we provide a comparison of the angular Rhines scale for Jupiter, an ordinary hot Jupiter, and a RGHJ. Dynamical models suggest that wind speeds in hot Jupiter atmospheres are roughly a kilometer per second (Showman et al. 2008; Rauscher \& Menou 2009; Heng et al. 2011, and many others). These extremely fast winds are largely driven by the strong day-night temperature contrast imposed by tidally locked rotation, and tend to be organized into large-scale jets (typical angular Rhines scale of $\sim 100^{\circ}$ ). A RGHJ, lacking this strong temperature contrast, might have significantly slower winds. In Table 1, we have assumed $100 \mathrm{~m} \mathrm{~s}^{-1}$ as an illustrative value. Since the angular Rhines scale depends on wind speed to the $1 / 2$ power, errors of order unity in wind speed do not change the qualitative conclusion that the combination of faster rotation and slower winds would lead the general character of atmospheric dynamics on a RGHJ to be more similar to that of Jupiter, with many narrow banded jets.

\section{CONCLUSIONS}

It has long been appreciated that as a star expands and grows more luminous after its mainsequence lifetime, the orbital radii corresponding to a given equilibrium temperature move farther out from the star. Lopez et al. (2005) and Schröder \& Connon Smith (2008), for instance, considered the outward expansion of the habitable zone after a star leaves the main sequence, although whether life would be likely to have enough time to develop from abiotic conditions in the limited time available remains an open question (Spiegel \& Turner 2012). A large number of known exoplanets are far enough from their stars that they are not currently strongly irradiated but they will be in the future. To investigate the properties that such planetary companions will have in the future, when their stars are much more luminous, we computed a suite of post-main-sequence stellar models to see how far out an object could be and still plausibly be called a hot Jupiter around a sufficiently evolved star. We found that, as far out as $\sim 35 \mathrm{AU}$ from a $3-M_{\odot}$ star, a companion might transiently be highly enough irradiated to merit the moniker "hot Jupiter." We argued that massive planets might accrete an amount of stellar wind that is large compared with their own atmospheres, resulting in significant atmospheric pollution if the stellar wind's composition is different from the planet's, and perhaps creating the atmospheric signature of a CRP (even if the planet's bulk composition is not carbon-rich). We showed that an angular-Rhinesscale argument suggests that that RGHJs might be expected to have narrow-banded atmospheric jets, in contrast to main-sequence hot Jupiters.

Detection of RGHJs might prove to be observationally challenging. The probability that a planet transits its star increases dramatically as the star's radius increases by a factor of 100 or more, but recognizing transits (or occultations) of RGHJs could prove nearly impossible, since the transit depth becomes tiny $\left(\sim 10^{-6}\right)$ and the duration could last weeks to months (Assef et al. 2009). High-contrast imaging is a potential avenue for detecting photons from RGHJs, but the solar neighborhood is not rich in likely suitable targets and the required angular resolution to resolve companions to distant giant stars might be daunting, given present technology. It might actually be easier to find evidence of former RGHJs than of current RGHJs, perhaps by surveying white dwarfs for companions at several AU (Gould \& Kilic 2008). Purely because of the timescale of photon diffusion, energetic arguments suggest that a companion should remain hot, after an AGB star fades into oblivion, for roughly as long as it had been highly irradiated (a few hundred million years). Such a reheated companion, perhaps identified by its infrared excess relative to the white dwarf's spectral energy distribution, might be mistaken for a more massive companion. However, this potential source of confusion is quantitatively significant only for very young white dwarfs, since the thermal relaxation timescale of a reheated planet is also of order a few hundred million years, short in comparison to a Hubble time.

The searches for exoplanets in the last two decades have uncovered many surprises. Here, we investigated the properties of a category of giant planets that might be found soon and that have 
not been studied in detail previously. Hot Jupiters around post-main-sequence stars must exist in the Galaxy and represent a future evolutionary stage of many of the known jovian-mass planets, including our own Jupiter.

\section{ACKNOWLEDGMENTS}

We thank many people for useful discussions, in particular Rodrigo Fernandez, JJ Hermes, Ruobing Dong, Scott Tremaine, Ed Turner, Jason Nordhaus, Adam Burrows, Jeremy Goodman, Doug Lin, Subo Dong, Jay Farihi, Jonathan Mitchell, Adam Showman, Kristen Menou, Scott Gaudi, and John Johnson. We also thank an anonymous referee for helpful comments that materially improved the manuscript. DSS gratefully acknowledges support from NSF grant AST-0807444 and the Keck Fellowship. NM acknowledges support from the Yale Center for Astronomy and Astrophysics through the YCAA postdoctoral Fellowship.

\section{REFERENCES}

Artigau, É., Bouchard, S., Doyon, R., \& Lafrenière, D. 2009, ApJ, 701, 1534

Asplund, M., Grevesse, N., Sauval, A. J., \& Scott, P. 2009, ARA\&A, 47, 481

Assef, R. J., Gaudi, B. S., \& Stanek, K. Z. 2009, ApJ, 701, 1616

Atreya, S. K. 2004, in ESA Special Publication, Vol. 544, Planetary Probe Atmospheric Entry and Descent Trajectory Analysis and Science, ed. A. Wilson, 57-62

Batygin, K. \& Stevenson, D. J. 2010, ApJ, 714, L238

Batygin, K., Stevenson, D. J., \& Bodenheimer, P. H. 2011, ArXiv e-prints

Bolton, S. J. \& Bolton. 2010, in IAU Symposium, Vol. 269, IAU Symposium, 92-100

Bondi, H. \& Hoyle, F. 1944, MNRAS, 104, 273

Burrows, A. \& Sharp, C. M. 1999, ApJ, 512, 843

Burrows, A., Sudarsky, D., \& Hubeny, I. 2006, ApJ, 650, 1140

Carlberg, J. K., Majewski, S. R., \& Arras, P. 2009, ApJ, 700,832

Charpinet, S., Fontaine, G., Brassard, P., Green, E. M., van Grootel, V., Randall, S. K., Silvotti, R., Baran, A. S., Østensen, R. H., Kawaler, S. D., \& Telting, J. H. 2011, Nature, 480, 496

Clarke, F. J., Tinney, C. G., \& Covey, K. R. 2002, MNRAS, 332, 361

Dong, R., Wang, Y., Lin, D. N. C., \& Liu, X.-W. 2010, ApJ, 715, 1036

Farihi, J., Hoard, D. W., \& Wachter, S. 2010, ApJS, 190, 275

Farihi, J., Subasavage, J. P., Nelan, E. P., Harris, H. C., Dahn, C. C., Nordhaus, J., \& Spiegel, D. S. 2012, ArXiv e-prints

Fortney, J. J., Marley, M. S., Saumon, D., \& Lodders, K. 2008, ApJ, 683, 1104

Gould, A. \& Kilic, M. 2008, ApJ, 673, L75

Guillot, T. 2010, A\&A, 520, A27+

Guillot, T. \& Showman, A. P. 2002, A\&A, 385, 156

Hebb, L., Collier-Cameron, A., Loeillet, B., Pollacco, D., Hébrard, G., Street, R. A., Bouchy, F., Stempels, H. C. Moutou, C., Simpson, E., Udry, S., Joshi, Y. C., West, R. G., Skillen, I., Wilson, D. M., McDonald, I., Gibson, N. P., Aigrain, S., Anderson, D. R., Benn, C. R., Christian, D. J., Enoch, B., Haswell, C. A., Hellier, C., Horne, K., Irwin, J., Lister, T. A., Maxted, P., Mayor, M., Norton, A. J., Parley, N., Pont, F., Queloz, D., Smalley, B., \& Wheatley, P. J. 2009, ApJ, 693, 1920

Heng, K., Menou, K., \& Phillipps, P. J. 2011, MNRAS, 413, 2380

Hogan, E., Burleigh, M. R., \& Clarke, F. J. 2009, MNRAS, 396,2074

Hubeny, I. \& Burrows, A. 2007, ApJ, 669, 1248

Ibgui, L. \& Burrows, A. 2009, ApJ, 700, 1921
Johnson, J. A., Payne, M., Howard, A. W., Clubb, K. I., Ford, E. B., Bowler, B. P., Henry, G. W., Fischer, D. A., Marcy, G. W., Brewer, J. M., Schwab, C., Reffert, S., \& Lowe, T. B. 2011, AJ, 141, 16

Karkoschka, E. \& Tomasko, M. G. 2010, Icarus, 205, 674

Kippenhahn, R. \& Weigert, A. 1990, Stellar Structure and Evolution, ed. Kippenhahn, R. \& Weigert, A.

Kratter, K. M. \& perets, H. B. 2012, ArXiv e-prints

Le Bertre, T., Matsuura, M., Winters, J. M., Murakami, H., Yamamura, I., Freund, M., \& Tanaka, M. 2001, A\&A, 376,997

Liu, X., Burrows, A., \& Ibgui, L. 2008, ApJ, 687, 1191

Livio, M. \& Warner, B. 1984, The Observatory, 104, 152

Lloyd, J. P. 2011, ApJ, 739, L49

Lopez, B., Schneider, J., \& Danchi, W. C. 2005, ApJ, 627, 974

Madhusudhan, N., Harrington, J., Stevenson, K. B., Nymeyer, S., Campo, C. J., Wheatley, P. J., Deming, D., Blecic, J., Hardy, R. A., Lust, N. B., Anderson, D. R., Collier-Cameron, A., Britt, C. B. T., Bowman, W. C., Hebb, L., Hellier, C., Maxted, P. F. L., Pollacco, D., \& West, R. G. 2011a, Nature, 469, 64

Madhusudhan, N., Mousis, O., Johnson, T. V., \& Lunine, J. I. 2011b, ApJ, 743, 191

Madhusudhan, N. \& Seager, S. 2009, ApJ, 707, 24

—. 2011, ApJ, 729, 41

Marcy, G. W. \& Butler, R. P. 1996, ApJ, 464, L147+

Marley, M. S., Fortney, J. J., Hubickyj, O., Bodenheimer, P., \& Lissauer, J. J. 2007, ApJ, 655, 541

Maxted, P. F. L., Napiwotzki, R., Dobbie, P. D., \& Burleigh, M. R. 2006, Nature, 442, 543

Mayor, M. \& Queloz, D. 1995, Nature, 378, 355

Menou, K. 2012, ApJ, 745, 138

Miller-Ricci, E., Seager, S., \& Sasselov, D. 2009, ApJ, 690, 1056

Mullally, F., Kilic, M., Reach, W. T., Kuchner, M. J., von Hippel, T., Burrows, A., \& Winget, D. E. 2007, ApJS, 171,206

Mullally, F., Reach, W. T., De Gennaro, S., \& Burrows, A. 2009, ApJ, 694, 327

Mullally, F., Winget, D. E., De Gennaro, S., Jeffery, E., Thompson, S. E., Chandler, D., \& Kepler, S. O. 2008, ApJ, 676, 573

Nordhaus, J. \& Blackman, E. G. 2006, MNRAS, 370, 2004

Nordhaus, J., Blackman, E. G., \& Frank, A. 2007, MNRAS, 376,599

Nordhaus, J., Spiegel, D. S., Ibgui, L., Goodman, J., \& Burrows, A. 2010, MNRAS, 1164

Nordhaus, J., Wellons, S., Spiegel, D. S., Metzger, B. D., \& Blackman, E. G. 2011, Proceedings of the National Academy of Science, 108, 3135

Öberg, K. I., Murray-Clay, R., \& Bergin, E. A. 2011, ApJ, 743, L16

Paxton, B. 2004, PASP, 116, 699 
Perna, R., Menou, K., \& Rauscher, E. 2010a, ApJ, 719, 1421 -. 2010b, ApJ, 724, 313

Rauscher, E. \& Menou, K. 2009, ArXiv e-prints

Schneider, J., Dedieu, C., Le Sidaner, P., Savalle, R., \& Zolotukhin, I. 2011, ArXiv e-prints

Schröder, K.-P. \& Connon Smith, R. 2008, MNRAS, 386, 155

Seager, S., Richardson, L. J., Hansen, B. M. S., Menou, K., Cho, J. Y.-K., \& Deming, D. 2005, ApJ, 632, 1122

Showman, A. P., Cho, J. Y.-K., \& Menou, K. Atmospheric Circulation of Exoplanets, ed. Seager, S., 471-516

Showman, A. P., Fortney, J. J., Lian, Y., Marley, M. S., Freedman, R. S., Knutson, H. A., \& Charbonneau, D. 2008, ArXiv e-prints

Soker, N., Livio, M., \& Harpaz, A. 1984, MNRAS, 210, 189

Spiegel, D. S. \& Burrows, A. 2012, ApJ, 745, 174

Spiegel, D. S., Burrows, A., \& Milsom, J. A. 2011, ApJ, 727,57

Spiegel, D. S., Silverio, K., \& Burrows, A. 2009, ApJ, 699, 1487
Spiegel, D. S. \& Turner, E. L. 2012, Proceedings of the National Academy of Science, 109, 395

Struck-Marcell, C. 1988, ApJ, 330, 986

Vasavada, A. R. \& Showman, A. P. 2005, Reports on Progress in Physics, 68, 1935

Veras, D., Wyatt, M. C., Mustill, A. J., Bonsor, A., \& Eldridge, J. J. 2011, MNRAS, 417, 2104

Villaver, E. \& Livio, M. 2007, ApJ, 661, 1192

-. 2009, ApJ, 705, L81

Visscher, C. \& Moses, J. I. 2011, ApJ, 738, 72

Willson, L. A. 2000, ARA\&A, 38, 573

Wright, J. T., Fakhouri, O., Marcy, G. W., Han, E., Feng, Y., Johnson, J. A., Howard, A. W., Fischer, D. A., Valenti, J. A., Anderson, J., \& Piskunov, N. 2011, PASP, 123,412

Wu, Y. \& Lithwick, Y. 2012, ArXiv e-prints

Zendejas, J., Segura, A., \& Raga, A. C. 2010, Icarus, 210, 539

Zuckerman, B. \& Dyck, H. M. 1989, A\&A, 209, 119

Zuckerman, B., Melis, C., Klein, B., Koester, D., \& Jura, M. 2010, ApJ, 722, 725 